\title{
Anisotropic carrier and exciton confinement in T-shaped quantum wires revealed by magneto-photoluminescence
}

\author{
Langbein, Wolfgang Werner; Gislason, Hannes; Hvam, Jørn Märcher; Zeman, J
}

Published in:

Summaries of papers presented at the International Quantum Electronics Conference

Link to article, DOI:

10.1109/IQEC.1998.680177

Publication date:

1998

Document Version

Publisher's PDF, also known as Version of record

Link back to DTU Orbit

Citation (APA):

Langbein, W. W., Gislason, H., Hvam, J. M., \& Zeman, J. (1998). Anisotropic carrier and exciton confinement in T-shaped quantum wires revealed by magneto-photoluminescence. In Summaries of papers presented at the International Quantum Electronics Conference (pp. 86-87). IEEE. https://doi.org/10.1109/IQEC.1998.680177

\section{General rights}

Copyright and moral rights for the publications made accessible in the public portal are retained by the authors and/or other copyright owners and it is a condition of accessing publications that users recognise and abide by the legal requirements associated with these rights.

- Users may download and print one copy of any publication from the public portal for the purpose of private study or research.

- You may not further distribute the material or use it for any profit-making activity or commercial gain

- You may freely distribute the URL identifying the publication in the public portal 
Linköping University, S-581 83 Linköping, Sweden

1. D.A.B. Miller, D.S. Chemla, S. SchmittRink, Appl. Phys. Lett. 52, 2154 (1988),

2. C. Juang, C.Y. Chang, Appl. Phys. Lett. 58, 1527 (1991).

3. F. Vouilloz, D.Y. Oberli, M.-A. Dupertuis, A. Gustafsson, F. Reinhardt, E. Kapon, Phys. Rev. Lett. 78, 1580 (1997).

\section{QWA4}

9:00 am

Anisotropic carrier and exciton confinement in T-shaped quantum wires revealed by magneto-photoluminescence

W. Langbein, H. Gislason, J.M. Hvam, J. Zeman* Mikroelektronik Centret, The Technical University of Denmark, Building 345 east, DK-2800 Lyngby, Denmark; E-mail: langbein@mic.dtu.dk

The realization of one-dimensional (1D) semiconductor nanostructures with large confinement energies is of importance for device applications. Different techniques such as growth on tilted substrates (Serpentine superlattices) or prepatterned substrates (V-groove quantum wires) and the cleaved-edge overgrowth of T-shaped structures ${ }^{1,2}$ have heen demonstrated. For the T-shaped structures, the confinement energy has been recently increased to above $2 \mathrm{k}_{\mathrm{B}} \mathrm{T}$ at room temperature by optimizing structure parameters. ${ }^{3,4} \mathrm{~A}$ decreased exciton diameter in the [110] direction in the wire compared with the [110] well was revealed by magneto-photoluminescence. ${ }^{5}$ We determine here the extension of the T-shaped quantum wire (T-QWR) state in both confining directions $[110]$ and $[001]$ to verify its $1 D$ character, as shown for crescent-shaped wires. ${ }^{6,7}$

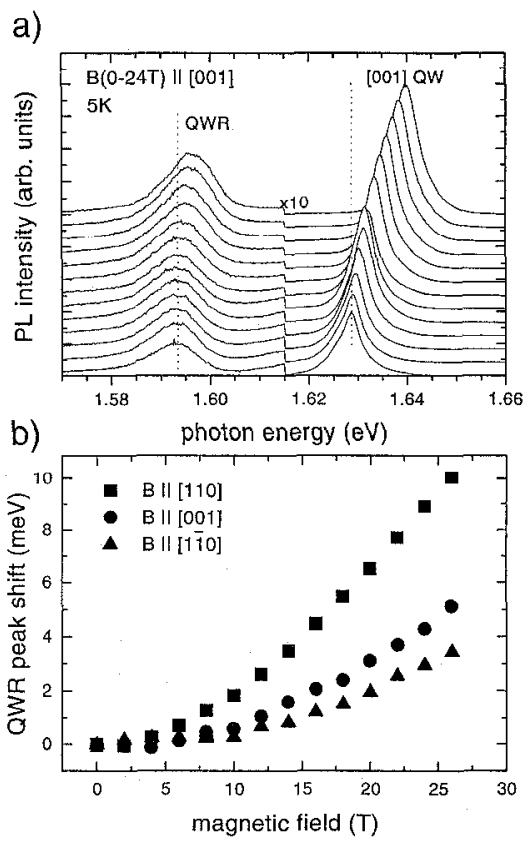

QWA4 Fig. 1. (a) QWR PL spectra for magnetic fields from 0 to $24 \mathrm{~T}$ in the [001] direction. The QWR and the [001] QW transitions are visible. (b) Energy shift of the QWR PL as a function of the field strength for field directions as labeled.
QWA4 Table 1. Deduced Parameters of Excitonic Transition of T-QWR Sample

\begin{tabular}{lcc}
\hline Property & Value & Model \\
\hline $\mathrm{a}_{\mathrm{B}}^{[110]}$ & $2.1 \mathrm{~nm}$ & \\
$\mathrm{a}_{\mathrm{B}}^{[001]}$ & $5.6 \mathrm{~nm}$ & anisotropic \\
$\mathrm{a}_{\mathrm{B}}^{[110]}$ & $10.2 \mathrm{~nm}$ & exciton \\
$\mathrm{E}_{\mathrm{B}}$ & $11 \mathrm{meV}$ & \\
$\mathrm{w}_{\mathrm{FWHM}}^{[110]}$ & $6.5 \mathrm{~nm}$ & confined \\
$\mathrm{w}_{\mathrm{FWHM}}^{[001]}$ & $10.3 \mathrm{~nm}$ & carrier \\
\hline
\end{tabular}

exciton model, 6 we can deduce the three exciton Bohr radii $\left(a_{\mathrm{B}}^{[001]}, a_{\mathrm{B}}^{[110]}\right.$, and $\left.\mathrm{a}_{\mathrm{B}}^{[1 \overline{1} 0]}\right)$ and the exciton binding energy $\mathrm{E}_{\mathrm{B}}$ using the diamagnetic shifts (see Table 1). The result shows an anisotropic exciton wave function and an exciton binding energy of $11 \mathrm{meV}$, in agreement with recent calculations. ${ }^{3,9}$

Using a free particle model, ${ }^{6,10}$ we can determine the extension $w_{\text {FWHM }}^{[001]}, w_{\text {FWHM }}^{[110]}$ of the electron wave function along the two confinement directions [001] and [110] (see Table 1).
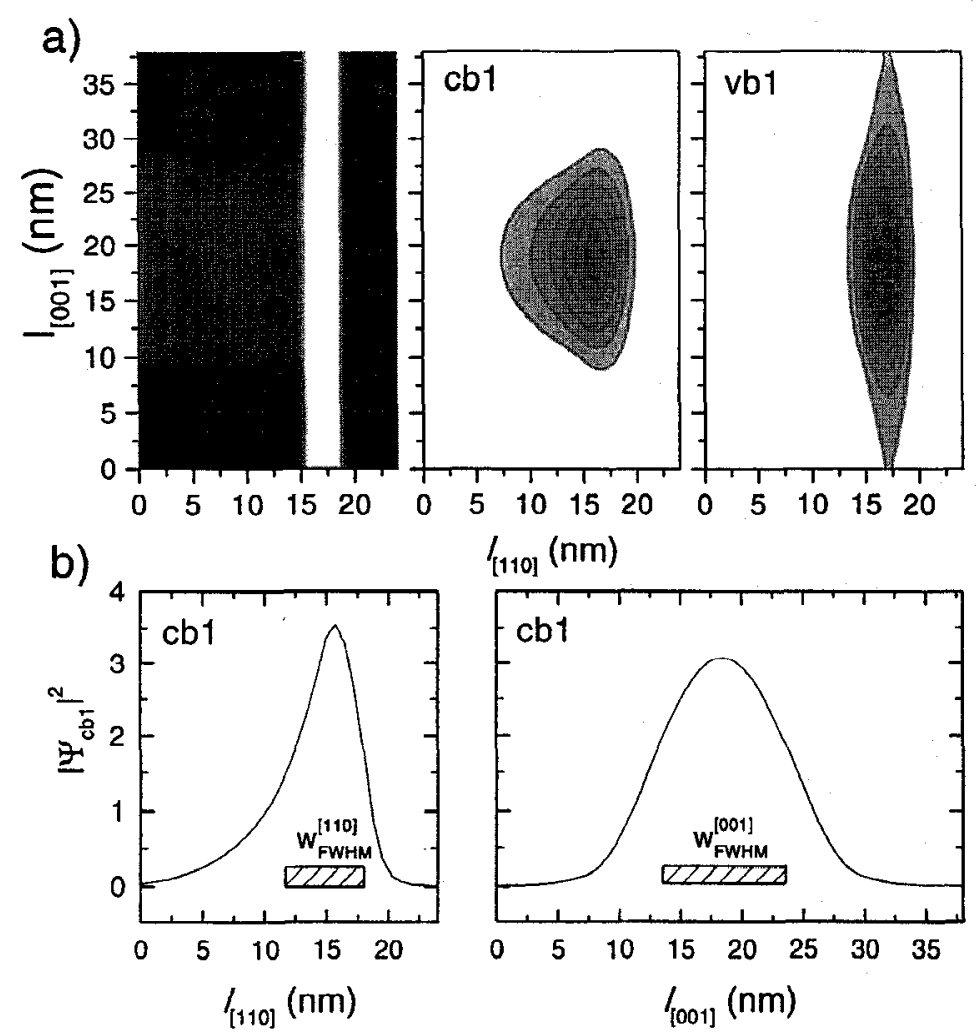

QWA4 Fig. 2. (a) Calculated QWR states in the T-QWR structure. The structure shape is shown on the left; the contours of constant probability are plotted in the middle and right graphs for the conduction and valence band state, respectively. (b) Calculated probability distributions of the conduction band state averaged along the [001] and [110] direction, respectively. The bars give the measured extensions $\mathrm{w}_{\mathrm{FWHM}}^{[001]}, \mathrm{w}_{\mathrm{FWHM}}^{[110]}$.

The investigated optimized T-QWR sample with $37 \mathrm{meV}$ confinement energy consists of a 18-nm [001] $\mathrm{Al}_{0.09} \mathrm{Ga}_{0.91}$ As multiple quantum well (QW) structure intersecting with a 4.3-nm [110] GaAs QW, within $\mathrm{Al}_{0.3} \mathrm{Ga}_{0.7} \mathrm{As}$ barriers. ${ }^{8}$ It was excited by an $\mathrm{Ar}^{+}$laser at 514 nm using a multimode fiber. The photoluminescence (PL) at temperatures between $5 \mathrm{~K}$ and $30 \mathrm{~K}$ was collected by the fiber, dispersed in a monochromator, and detected by a gallium aresenide (GaAs) photomultiplier. A magnetic field from 0 to $26 \mathrm{~T}$ was applied by a superconducting and a resistive magnet.

The PL spectra for the field direction [001] are shown in Fig. 1a. It is seen that the T-QWR PL shows a smaller shift to high energies with increasing field than the [001] QW PL. The shift of the T-QWR PL is shown in Fig. Ib for the three principal directions of the field. It shows three distinctly different magnitudes, according to the different projected sizes of the QWR exciton state.

To quantitatively analyze the data, we apply two different models. Using an anisotropic 3D
In Fig. $2 \mathrm{a}$, the calculated confined electron and hole squared wave functions for the QWR $1^{3}$ are shown, localized at the intersection. Taking these results, the calculated probability distributions along the two quantization directions [001] and [110] are compared with the deduced FWHM of the electron wave functions in Fig. 2b. A quantitative agreement is found.

This work was supported by TMR contract ERBFMGECT 950077 , and by CNAST.

${ }^{*}$ Grenoble High Magnetic Field Laboratory CNRS/MPIF, Avenue des Martyrs, F-38042 Grenoble, France

1. L. Pfeiffer et al., J. Crystal Growth 127, 849 (1993).

2. T. Someya, H. Akiyama, H. Sakaki, J. Appl. Phys. 79, 2522 (1996).

3. W. Langbein, H. Gislason, J.M. Hvam, Phys. Rev. B 54, 14595 (1996).

4. H. Gislason, W. Langbein, J.M. Hvam, Appl. Phys. Lett. 69, 3248 (1996).

5. T. Someya, H. Akiyama, H. Sakaki, Phys. Rev. Lett. 74, 3664 (1995). 
6. Y. Nagamune et al., Phys. Rev. Lett. 69, 2963 (1992).

7. Y. Nagamune et al., Appl. Phys. Lett. 66, 2502 (1995)

8. H. Gislason, C. Sørensen, J.M. Hvam, Appl. Phys. Lett. 69, 800 (1996).

9. F. Rossi, G. Goldoni, E. Molinari, Phys. Rev. Lett. 78, 3527 (1997).

10. T. Tanaka, Y. Arakawa, G.W.E. Bauer, Phys. Rev. B 50, 7719 (1994).

\section{QWA5}

9:15 am

Carrier transport in a single GaAs quantum wire structure studied by timeresolved near-field spectroscopy

A. Richter, M. Süptitz, Ch. Lienau, T. Elsaesser, M. Ramsteiner, ${ }^{*}$ R. Nötzel, ${ }^{*}$ K.H. Ploog* Max-Born-Institut für Nichtlineare Optik und Kurzzeitspektroskopie, Rudower Chaussee 6, D-12489 Berlin, Germany; E-mail: lienau@mbi.fta-berlin.de

Direct studies of the transport of photogenerated electron-hole pairs in semiconductor nanostructures require both high spatial and temporal resolution. Near-field scanning optical microscopy (NSOM), offering subwavelength spatial resolution in the $100-\mathrm{nm}$ range in combination with time-ressolved detection schemes is a particularly promising technique for such experiments. Here we report on the first time-resolved near-field spectroscopic investigation of the carrier dynamics in single gallium arsenide $(\mathrm{GaAs}) /(\mathrm{AlGa}) \mathrm{As}$ sidewall quantum wires.

Quantum wires (QWR) with a lateral width of $60 \mathrm{~nm}$ and a thickness of up to $13 \mathrm{~nm}$ embedded by a $6-\mathrm{nm}$ GaAs quantum well (QW) were grown by molecular beam epitaxy of $\mathrm{GaAs} /(\mathrm{AlGa})$ As multilayer structures on patterned GaAs (311)A substrates along the sidewall of 15- to 20 -nm-high mesa stripes oriented along the [01-1] direction (Fig. 1a). ${ }^{1}$
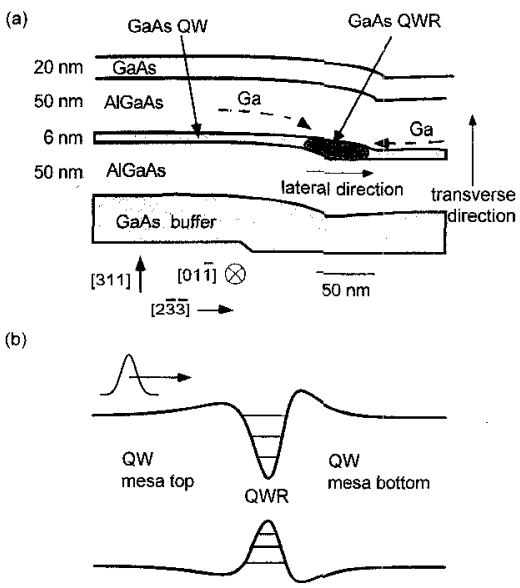

QWA5 Fig. 1. (a) Schematic of the quantum wire (QWR) structure. The thickness variation of the GaAs quantum well along the lateral [2-3-3] direction leads to a quasi one-dimensional confinement. (b) Schematic lateral confinement potential and subband structure. The QWR is surrounded by shallow asymmetric energetic barriers with a height about $18 \mathrm{meV}$ on the mesa top and $14 \mathrm{meV}$ on the mesa bottom.
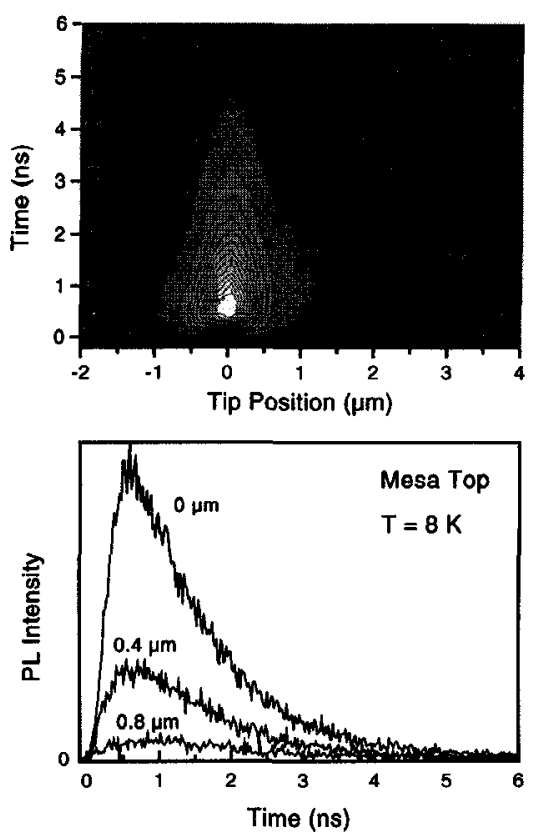

QWA5 Fig. 2. (a) Time-resolved QWR luminescence for spatially resolved excitation of the excitonic absorption band of the GaAs QW through the near-field probe tip as a function of tip position along the lateral axis at a sample temperature of $10 \mathrm{~K}$. White regions are regions of high photoluminescence at the detection wavelength. (b) Cross sections through Fig. 2a at fixed excitation positions. Trapping of carriers generated within the flat area QW is strongly suppressed.

The confinement potential of the QWR and the embedding $\mathrm{QW}$ as directly extracted from NSOM photoluminescence excitation (PLE) spectra at $77 \mathrm{~K},{ }^{2}$ Fig. $1 \mathrm{~b}$, reveals shallow asymmetric energetic barriers with a height of about $18 \mathrm{meV}$ on the mesa top and $14 \mathrm{meV}$ on the mesa bottom in the vicinity of the QWR.

In our experiments, electron-hole pairs are created by transmitting a fs-Ti:Sapphire laser pulse at $1.614 \mathrm{eV}$ through an NSOM fiber probe with an aperture diameter of about 200 $\mathrm{nm}$. The laser spectrum overlaps with the excitonic absorption band of the GaAs QW. QWR luminescence at $1.54 \mathrm{eV}$ is detected in the farfield as a function of tip position using timecorrelated single-photon counting as the tip is scanned perpendicularly to the QWR.

At a sample temperature of $10 \mathrm{~K}$, QWR luminescence is observed for localized excitation in a narrow region around the QWR, in between the two energetic barriers separated by about $800 \mathrm{~nm}$. In this region, the capture into the QWR and the energetic relaxation within the QWR is fast, so that with the temporal resolution of our experiment of $250 \mathrm{ps,}$ the temporal dependence of the QWR luminescence is dominated by the QWR recombination lifetime of $0.9 \mathrm{~ns}$, independent of excitation position. For excitation outside the barriers, the diffusive transport of excitons across the barriers is strongly suppressed and trapping of carriers generated within the flat area QW is prohibited.

At a temperature of $100 \mathrm{~K}$, a drastically different behavior is found. QWR luminescence is observed for QW excitation at dis-
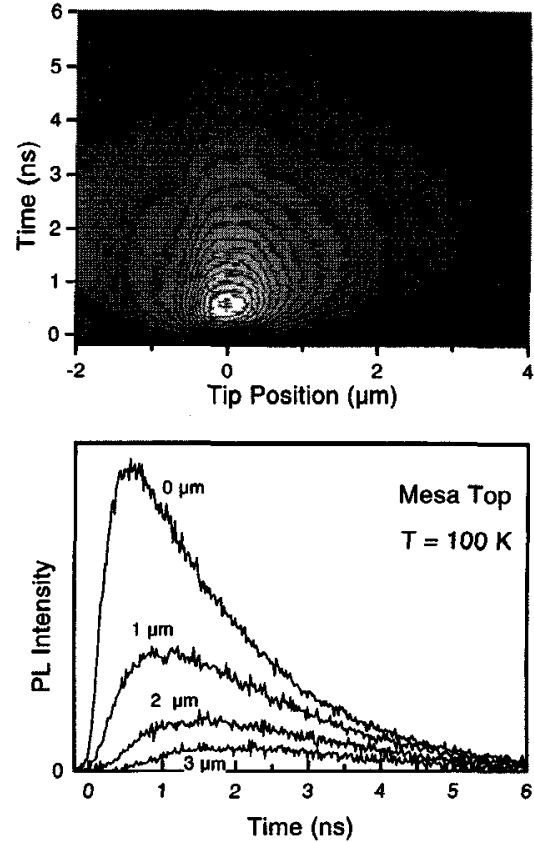

QWA5 Fig. 3. (a) Time-resolved QWR luminescence at a sample temperature of $100 \mathrm{~K}$. Experimental conditions as in Fig. 2. (b) Cross sections through Fig. $3 a$ at fixed excitation positions. The time-resolved data directly reveal the delayed onset of QWR luminescence for excitation positions between 1 and $3 \mu \mathrm{m}$.

tances of several micrometers away from the QWR. This occurrence of QWR luminescence involves ambipolar drift-diffusion across the barriers. The time-resolved data directly reveal the delayed onset of QWR luminescence for excitation positions between 0.5 and $3 \mu \mathrm{m}$.

The experiments directly demonstrate the pronounced influence of spatial bandgap variations on a subwavelength scale on the real-space carrier transfer within the two-dimensional (2D) continuum and thus on the carrier trapping dynamics into the $1 \mathrm{D}$ QWR states.

*Paul-Drude-Institut für Festkörperelektronik, Hausvoigteiplatz 5-7, D-10117 Berlin, Germany

1. R. Nötzel, J. Menniger, M. Ramsteiner, A. Ruiz, H.P. Schönherr, K.H. Ploog, Appl. Phys. Lett. 68, 1132 (1996).

2. A. Richter, G. Behme, M. Süptitz, Ch. Lienau, T. Elsaesser, M. Ramsteiner, R. Nötzel, K.H. Ploog, Phys. Rev. Lett. 79, 2145 (1997)

9:30 am

Large third-order optical nonlinearity in

Au:dielectric composite films in femtosecond time scale

H.B. Liao, R.F. Xiao, H. Wang, K.S. Wong, G.K.L. Wong, Department of Physics, Hong Kong University of Science \& Technology, Clear Water Bay, Kowloon, Hong Kong

We report here the realization of large thirdorder nonlinear susceptibility, $\chi^{(3)}$, from Au:dielectric $\left(\mathrm{SiO}_{2}\right.$ and $\mathrm{TiO}_{2}$ ) composite films near the Au percolation threshold. The composite films were prepared by co-sputtering 